\title{
Complexity, Dynamics, Control, and Applications of Nonlinear Systems with Multistability
}

\author{
Viet-Thanh Pham $\mathbb{D}^{1,2,3}$ Sundarapandian Vaidyanathan ${ }^{1}{ }^{4}{ }^{4}$ and Tomasz Kapitaniak ${ }^{3}$ \\ ${ }^{1}$ Faculty of Electrical and Electronic Engineering, Phenikaa Institute for Advanced Study (PIAS), Phenikaa University, Yen Nghia, \\ Ha Dong District, Hanoi 100000, Vietnam \\ ${ }^{2}$ Phenikaa Research and Technology Institute (PRATI), A\&A Green Phoenix Group, 167 Hoang Ngan, Hanoi 100000, Vietnam \\ ${ }^{3}$ Division of Dynamics, Lodz University of Technology, Stefanowskiego 1/15, Lodz 90-924, Poland \\ ${ }^{4}$ Research and Development Centre, Vel Tech University, No. 42, Avadi-Vel Tech Road, Avadi, Chennai, \\ Tamil Nadu 600062, India
}

Correspondence should be addressed to Viet-Thanh Pham; thanh.phamviet@phenikaa-uni.edu.vn

Received 4 June 2020; Accepted 5 June 2020; Published 4 September 2020

Copyright (c) 2020 Viet-Thanh Pham et al. This is an open access article distributed under the Creative Commons Attribution License, which permits unrestricted use, distribution, and reproduction in any medium, provided the original work is properly cited.

Multistability is a critical property of nonlinear dynamical systems, where a variety of phenomena such as coexisting attractors can appear for the same parameters but with different initial conditions. The flexibility in the system's performance can be achieved without changing parameters. Complex dynamics have been observed in multistable systems, and we have witnessed systems with multistability in numerous fields ranging across physics, biology, chemistry, electronics, and mechanics, as well as reported applications in oscillators and secure communications. It is now well established from a variety of studies that multistable systems are very sensitive to both random noise and perturbations. Numerous studies such as open-loop control, feedback control, adaptive control, intelligent control, and stochastic control have been attempted to control multistable systems.

Recent attention has focused more on extraordinary cases of systems with multistability, such as systems with megastability and extreme multistability. A megastable system can display countably infinite number of coexisting attractors, whereas an extreme multistable system can exhibit an uncountably infinite number of coexisting attractors. However, there are still various theoretical and technical issues which should be investigated in such multistable systems. Circuit design (numerical and hardware) of multistable systems is a related research problem with real-world applications, and fractional-order modelling and realization of multistable systems also constitute a complex and challenging task. Furthermore, circuit realizations (simulations and hardware design) of multistable systems are useful for various practical applications in engineering.

This special issue aims to introduce and discuss novel results, control techniques, and circuit simulations for complex nonlinear systems with multistability. We had received a total of 94 submissions. After the review process, the acceptance rate is approximately $35.1 \%$. This special issue contains 33 articles, the contents of which are summarized as follows.

In the article by M. F. Tsotsop et al., a new elegant hyperjerk system with three equilibria and hyperbolic sine nonlinearity is investigated. In contrast to other models of hyperjerk systems where either hidden or self-excited attractors are obtained, the case reported in this work represents a unique one which displays the coexistence of self-excited chaotic attractors and stable fixed points. The dynamic properties of the new system are explored in terms of equilibrium point analyses, symmetry, and dissipation and existence of attractors as well. Common analysis tools (i.e., bifurcation diagram, Lyapunov exponents, phase portrait, etc.) are used to highlight some important phenomena such as period-doubling bifurcation, chaos, periodic windows, and symmetric restoring crises. More interestingly, the system under consideration shows the coexistence of several types of stable states, including the 
coexistence of two, three, four, six, eight, and ten coexisting attractors. In addition, the system is shown to display antimonotonicity and offset boosting. Laboratory experimental measurements show very good coherence with the theoretical predictions.

The objective of the article by J. H. Pérez-Cruz is to estimate the unmeasurable variables of a multistable chaotic system using a Luenberger-like observer. First, the observability of the chaotic system is analyzed. Next, a Lipschitz constant is determined on the attractor of this system. Then, the methodology proposed by Raghavan and the result proposed by Thau are used to try to find an observer. Both attempts are unsuccessful. In spite of this, a Luenberger-like observer can still be used based on a proposed gain. The performance of this observer is tested by numerical simulation showing the convergence to zero of the estimation error. Finally, the chaotic system and its observer are implemented using 32-bit microcontrollers. The experimental results confirm good agreement between the responses of the implemented and simulated observers.

Semitensor product theory can deal with matrix multiplication with different number of columns and rows. Therefore, a new chaotic system for different high dimensions can be created by employing a semitensor product of chaotic systems with different dimensions so that more channels can be selected for encryption. R. Wang et al. propose a new chaotic system generated by the semitensor product applied on Qi and Lorenz systems. The corresponding dynamic characteristics of the new system are discussed in this article to verify the existences of different attractors. The detailed algorithm is illustrated in this article. The FPGA hardware encryption implementations are also elaborated and conducted. Correspondingly, the randomness tests are realized as well, and compared with those of the individual Qi system and Lorenz system, the proposed system in this article owns the better randomness characteristic. The statistical analyses, differential analyses, and correlation analyses are also discussed.

The article by M. Yao et al. focuses on power generation and nonlinear dynamic behaviors on a new bistable piezoelectric-electromagnetic energy harvester. Three different kinds of piezoelectric cantilever beam structures, which include the monostable piezoelectric cantilever beam, the bistable piezoelectric cantilever beam with the spring and the magnet, and the bistable piezoelectric cantilever beam with the spring, magnet and coil, are designed. The power generation efficiency and dynamic behaviors for each structure are experimentally studied, respectively. Due to the spring introduced, the system easily goes through the potential barrier. Experimental results show that the power generation structure of the bistable piezoelectric-electromagnetic harvester can vibrate between two steady states in a wider range of the frequency. Therefore, the effective frequency bandwidth is broadened about $2 \mathrm{~Hz}$ when the spring is introduced under the condition of the suitable magnetic distance. Comparing with the power generation efficiency for three different kinds of structures, it is found that the bistable piezoelectric-electromagnetic harvester has the optimum characteristics, which include the optimal magnetic distance of $15 \mathrm{~mm}$, the optimal load of $8 \mathrm{M} \Omega$, and the parameter variation law of coils. For this structure, the influences of the external excitation and the magnetic distance on the output voltage and dynamic behaviors of the system are examined.

In the article by $\mathrm{X}$. Liu et al., a new fractional-order discrete noninvertible map of cubic type is presented. Firstly, the stability of the equilibrium points for the map is examined. Secondly, the dynamics of the map with two different initial conditions are studied by numerical simulation when a parameter or a derivative order is varied. A series of attractors are displayed in various forms of periodic and chaotic ones. Furthermore, bifurcations with the simultaneous variation of both the parameter and the order are also analyzed in the three-dimensional space. Interior crises are found in the map as a parameter or an order varies. Thirdly, based on the stability theory of fractional-order discrete maps, a stabilization controller is proposed to control the chaos of the map, and the asymptotic convergence of the state variables is determined. Finally, the synchronization between the proposed map and the fractional-order discrete Loren map is investigated. Numerical simulations are used to verify the effectiveness of the designed synchronization controllers.

Aiming at the problem of weak security of compressed sensing, J. Yu et al. combine the cryptographic characteristics of chaotic systems with compressed sensing technology. In the actual research process, the existing image encryption technology needs to be applied to hardware. This article focuses on the combination of image encryption based on compressed sensing and digital logic circuits. The authors propose a novel technology of parallel image encryption based on a sequence generator. It uses a threedimensional chaotic map with multiple stability to generate a measurement matrix. This study also analyzes the effectiveness, reliability, and security of the parallel encryption algorithm for source noise pollution with different distribution characteristics. Simulation results show that parallel encryption technology can effectively improve the efficiency of information transmission and greatly enhance its security through key space expansion.

In the article by $\mathrm{X}$. Hu and P. Zhou, a 3D multistability chaotic system with two coexisting conditional symmetric attractors is studied by using a circuit block diagram and realized by using an electronic circuit. The simulation results show that two coexisting conditional symmetric attractors are emerged in this electronic circuit. Furthermore, synchronization of this 3D multistability chaotic system and its electronic circuit is studied. It shows that the linear resistor and the linear capacitor in parallel coupling can achieve synchronization in this chaotic electronic circuit. That is, the output voltage of the chaotic electronic circuit is coupled via one linear resistor and one linear capacitor in parallel coupling. The simulation results verify that synchronization of the chaotic electronic circuit can be achieved.

In the article by J. Zhang and $\mathrm{X}$. Xi, a decision-making competition game model concerning governments, agricultural enterprises, and the public, all of which participate in the reduction of nitrogen emissions in the watersheds, is established based on bounded rationality. First, the stability 
conditions of the equilibrium points in the system are discussed, and the stable region of the Nash equilibrium is determined. Then, the bifurcation diagram, maximal Lyapunov exponent, strange attractor, and sensitive dependence on the initial conditions are shown through numerical simulations. The research shows that the adjustment speed of three players' decisions may alter the stability of the Nash equilibrium point and lead to chaos in the system. Among these decisions, a government's decision has the largest effect on the system. In addition, authors found that some parameters will affect the stability of the system; when the parameters become beneficial for enterprises to reduce nitrogen emissions, the increase in the parameters can help control the chaotic market. Finally, the delay feedback control method is used to successfully control the chaos in the system and stabilize it at the Nash equilibrium point. The research of this article is of great significance to the environmental governance decisions and nitrogen reduction management.

L. Huang et al. have constructed a new 4D memristorbased chaotic system by using a smooth flux-controlled memristor to replace a resistor in the realization circuit of a 3D chaotic system. Compared with general chaotic systems, the chaotic system can generate many coexisting attractors. The proposed chaotic system not only possesses heterogeneous multistability but also possesses homogenous multistability. When the parameters of the system are fixed, the chaotic system only generates two kinds of chaotic attractors with different positions in a very large range of initial values. Different from other chaotic systems with continuous bifurcation diagrams, this system has discrete bifurcation diagrams when the initial values change. In addition, this article reveals the relationship between the symmetry of coexisting attractors and the symmetry of initial values in the system. The dynamic behaviors of the new system are analyzed by the equilibrium point and stability, bifurcation diagrams, Lyapunov exponents, and phase orbit diagrams. Finally, the chaotic attractors are captured through circuit simulation, which verifies numerical simulation.

L. K. Kengne et al. investigate the dynamics of a simple jerk system with a hyperbolic tangent whose symmetry is broken by adding a constant term modelling an external excitation force. They demonstrate that the modified system experiences several unusual and striking nonlinear phenomena including coexisting bifurcation branches, hysteretic dynamics, coexisting asymmetric bubbles, critical transitions, and multiple (i.e., up to six) coexisting asymmetric attractors for some suitable ranges of system parameters. These features are highlighted by exploiting common nonlinear analysis tools such as graphs of the largest Lyapunov exponent, bifurcation diagrams, phase portraits, and basins of attraction. The control of multistability is investigated by using the method of linear augmentation. The authors demonstrate that the multistable system can be converted to a monostable state by smoothly adjusting the coupling parameter. The theoretical results are confirmed by performing a series of PSpice simulations based on an electronic analogue of the system.
The article by A. Letafat et al. introduces an energy management strategy (EMS) for a hybrid energy system (HES) of a ferry boat with the goal to optimize the performance and reduce the operation cost. HES considered for the ferry boat consists of different devices such as the proton-exchange membrane fuel cell (PEMFC), LI-ION battery bank, and cold ironing (CI). PEMFC systems are appropriate to employ as they are not polluting. The battery bank compensates for the abrupt variations of the load as the fuel cell has a slow dynamic against sudden changes of the load. Also, CI systems can improve the reduction of the expenses of energy management, during hours where the ferry boat is located at the harbor. To study the performance, the cost, and the pollution contribution $\left(\mathrm{CO}_{2}, \mathrm{NO}_{X}\right.$, and $\mathrm{SO}_{X}$ ) of the proposed hybrid energy management strategy (HEMS), we compare it against three various types of HEM from the state-of-the-art and also available rule-based methods in the literature. The analysis results show a high applicability of the proposed HES. All results in this article have been obtained in the MATLAB software environment.

The article by R. J. Escalante-González and E. Campos presents an approach to design a multistable system with the one-directional (1D), two-directional (2D), and three-directional (3D) hidden multiscroll attractor by defining a vector field on $\mathbb{R}^{3}$ with an even number of equilibria. The design of multistable systems with hidden attractors remains a challenging task. Current design approaches are not as flexible as those that focus on self-excited attractors. To facilitate a design of hidden multiscroll attractors, they propose an approach that is based on the existence of selfexcited double-scroll attractors and switching surfaces whose relationship with the local manifolds associated to the equilibria leads to the appearance of the hidden attractor. The multistable systems produced by the approach could be explored for potential applications in cryptography since the number of attractors can be increased by design in multiple directions while preserving the hidden attractor allowing a bigger key space.

In the article by C. Dai et al., a heterogeneous diffusive prey-predator system is first proposed and then studied analytically and numerically. Some sufficient conditions are derived, including permanence and extinction of the system and the boundedness of the solution. The existence of the periodic solution and its stability are discussed as well. Furthermore, numerical results indicate that both the spatial heterogeneity and the time-periodic environment can influence the permanence and extinction of the system directly. The numerical results are consistent with the analytical analysis.

A. K. Tarboush and Z. Zhang investigate the impact of a periodically evolving domain on the dynamics of the diffusive West Nile virus. A reaction-diffusion model on a periodically and isotropically evolving domain which describes the transmission of the West Nile virus is proposed. In addition to the classical basic reproduction number, the spatial-temporal basic reproduction number depending on the periodic evolution rate is introduced, and its properties are discussed. Under some conditions, they explore the longtime behavior of the virus. The virus will go extinct if the 
spatial-temporal basic reproduction number is less than or equal to one. The persistence of the virus happens if the spatial-temporal basic reproduction number is greater than one. They consider a special case when the periodic evolution rate is equivalent to one to better understand the impact of the periodic evolution rate on the persistence or extinction of the virus. Some numerical simulations are performed in order to illustrate the analytical results. The theoretical analysis and numerical simulations show that the periodic change of the habitat range plays an important role in the West Nile virus transmission; in particular, the increase in periodic evolution rate has a positive effect on the spread of the virus.

The stability of grazing bifurcation is lost in three ways through the local analysis of the near-grazing dynamics using the classical concept of discontinuity mappings in the two-degree-of-freedom vibroimpact system with symmetrical constraints. For this instability problem, Z. Wang et al. presented a control strategy for the stability of grazing bifurcation by controlling the persistence of local attractors near the grazing trajectory in this vibroimpact system with symmetrical constraints. Discrete-in-time feedback controllers designed on two Poincare sections are employed to retain the existence of an attractor near the grazing trajectory. The implementation relies on the stability criterion under which a local attractor persists near a grazing trajectory. Based on the stability criterion, the control region of the two parameters is obtained, and the control strategy for the persistence of near-grazing attractors is designed accordingly. Especially, the chaos near codimension-two grazing bifurcation points was controlled by the control strategy. In the end, the results of numerical simulation are used to verify the feasibility of the control method.

In the article by L. Ge and S. Chen, a new data-driven learning method is investigated based on the dynamical data of the system. A regularized regression wavelet (RRW) approach is proposed to optimize the learning result for the system fault. Based on the optimizing results, a fault tolerant stability scheme is given. Then, the efficiency of the proposed technique is verified by a vertical take-off and landing (VTOL) aircraft stability example.

F. Yu et al. have introduced a novel multistable 5D memristive hyperchaotic system and its application. The interesting aspect of this chaotic system is that it has different types of coexisting attractors, chaos, hyperchaos, periods, and limit cycles. First, a novel 5D memristive hyperchaotic system is proposed by introducing a flux-controlled memristor with quadratic nonlinearity into an existing $4 \mathrm{D}$ fourwing chaotic system as a feedback term. Then, the phase portraits, Lyapunov exponential spectrum, bifurcation diagram, and spectral entropy are used to analyze the basic dynamics of the 5D memristive hyperchaotic system. For a specific set of parameters, we find an unusual metastability, which shows the transition from chaotic to periodic (period2 and period-3) dynamics. Moreover, its circuit implementation is also proposed. By using the chaoticity of the novel hyperchaotic system, they have developed a random number generator (RNG) for practical image encryption applications. Furthermore, security analyses are carried out with the RNG and image encryption designs.

The disk dynamo system, which is capable of chaotic behaviours, is obtained experimentally from two disk dynamos connected together. It models the geomagnetic field and is used to explain the reversals in its polarity. Actually, the parameters of the chaotic systems exhibit random fluctuation to a greater or lesser extent, which can carefully describe the disturbance made by environmental noise. In the article by $\mathrm{C}$. Feng et al., the global dynamics of the chaotic disk dynamo system with random fluctuating parameters are concerned, and some new results are presented. Based on the generalized Lyapunov function, the globally attractive and positive invariant set is given, including a twodimensional parabolic ultimate boundary and a four-dimensional ellipsoidal ultimate boundary. Furthermore, a set of sufficient conditions is derived for all solutions of the stochastic disk dynamo system being global convergent to the equilibrium point. Finally, numerical simulations are presented for verification.

In the article by F. Yu et al., multistable modified fourthorder autonomous Chua's chaotic system is investigated. In addition to the dynamic characteristics of third-order Chua's chaotic system itself, what interests authors is that this modified fourth-order autonomous Chua's chaotic system has five different types of coexisting attractors: double-scroll, single-band chaotic attractor, period-4 limit cycle, period-2 limit cycle, and period-1 limit cycle. Then, inductorless modified fourth-order autonomous Chua's chaotic circuit is proposed. The active elements as well as the synthetic inductor employed in this circuit are designed using secondgeneration current conveyors (CCIIs). The reason for using CCIIs is that they have high conversion rate and operation speed, which enable the circuit to work at a higher frequency range. The Multisim simulations confirm the theoretical estimates of the performance of the proposed circuit. Finally, using the RK-4 numerical algorithm of VHDL 32-bit IQMath floating-point number format, inductorless modified fourth-order autonomous Chua's chaotic system is implemented on FPGA for the development of embedded engineering applications based on chaos. The system is simulated and synthesized on the Virtex-6 FPGA chip. The maximum operating frequency of modified Chua's chaotic oscillator based on FPGA is $180.180 \mathrm{MHz}$. This study demonstrates that hardware-based multistable modified fourth-order autonomous Chua's chaotic system is a very good source of entropy and can be applied to various embedded systems based on chaos, including secure communication, cryptography, and random number generator.

J. Zhou and X. Chen construct a supply chain consisting of a manufacturer and a retailer. Considering channel integration and service cooperation, two dynamic Stackelberg game models are established: one without unit profit allocation $(M)$ and the other one with unit profit allocation $\left(M^{\varepsilon}\right)$. In two dynamic models, the authors analyze the influence of relevant parameters on the stability and complexity of the dynamic system and system profit by nonlinear system theory and numerical simulation. They found that the higher adjustment parameters can cause the system to 
lose stability, showing double-period bifurcation or waveshape chaos. The stable region becomes larger with increase in service value and value of unit profit sharing. Besides, when the system is in the chaotic state, the authors found that the profit of the system will fluctuate or even decline sharply; however, keeping the parameters in a certain range is helpful in maintaining the system stability and is conducive to decision makers to obtain steady profits. In order to control the chaos phenomenon, the state feedback method is employed to control the chaotic system well. This study provides some valuable significance to supply chain managers in channel integration and service cooperation.

Q. Li et al. consider a Stackelberg game model in a dualchannel supply chain, which is composed of a manufacturer and a retailer. The manufacturer and the retailer consider fairness concern in the market competition, and the manufacturer takes market share and profit as his/her business objectives. The entropy complexity and the dynamic characteristic of the dual-channel system are analyzed through mathematical analysis and numerical simulation, such as local stability, bifurcation, entropy, and chaos. The results show that, with the increase of price adjustment speed, the dual-channel supply chain is more complex and falls into a chaotic state in which system entropy increases; the stability of the dual-channel supply chain will be robust with the increase of weight of market share and weaken with the increase of the fairness concern level of the manufacturer and the retailer. The high level of fairness concern of the manufacturer and the retailer is always disadvantageous to the leading manufacturer but not always bad for the follower retailer. The performance of the dual-channel supply chain is improved with a high level of the manufacturer's fairness concern and reduced with a high level of the retailer's fairness concern. They also found that the retailer will gain more profits in the chaotic state than the stable state in the Stackelberg game model. The variable feedback control method is applied to control the chaos of the dual-channel supply chain, and choosing appropriate control parameters can make the dual-channel supply chain system return to the stable state from the chaotic state or delay the system to enter the bifurcation state. The research results can provide a guideline for enterprise decision-making.

In the article by B. Yan et al., dynamical complexity and multistability of electrostatic waves are investigated in fourcomponent homogenous and magnetized lunar wake plasma constituting beam electrons, heavier ions (alpha particles, $\mathrm{He}^{++}$), protons, and suprathermal electrons. The unperturbed dynamical system of the considered lunar wake plasma supports nonlinear and supernonlinear trajectories which correspond to nonlinear and supernonlinear electrostatic waves. On the contrary, the perturbed dynamical system of lunar wake plasma shows different types of coexisting attractors including periodic, quasi-periodic, and chaotic, investigated by phase plots and Lyapunov exponents. To confirm chaotic and nonchaotic dynamics in the perturbed lunar wake plasma, 0-1 chaos test is performed. Furthermore, a weighted recurrence-based entropy is implemented to investigate the dynamical complexity of the system. Numerical results show the existence of chaos with variation of complexity in the perturbed dynamics.

The Kadomtsev-Petviashvili equation is one of the wellstudied models of nonlinear waves in dispersive media and in multicomponent plasmas. In the article by H.-Y. Wu et al., the coupled Alice-Bob system of the Kadomtsev-Petviashvili equation is first constructed via the parity with a shift of the space variable $x$ and time reversal with a delay. By introducing an extended Bäcklund transformation, symmetry-breaking soliton, symmetry-breaking breather, and symmetry-breaking lump solutions for this system are presented through the established Hirota bilinear form. According to the corresponding constants in the involved ansatz function, a few fascinating symmetry-breaking structures of the presented explicit solutions are shown.

B. Feng is concerned with a linear thermoelastic laminated Timoshenko beam, where the heat conduction is given by Cattaneo's law. B. Feng firstly proves the global wellposedness of the system. For stability results, the author establishes exponential and polynomial stabilities by introducing a stability number $\chi$.

G. Kai et al. study the influence of two-delay feedback on the nonlinear dynamics behavior of the financial system, considering the linear stability of the equilibrium point under the condition of single delay and two delays. The system undergoes Hopf bifurcation near the equilibrium point. The stability and bifurcation directions of Hopf bifurcation are studied by using the normal form method and central manifold theory. The theoretical results are verified by numerical simulation. Furthermore, one feature of the proposed financial chaotic system is that its multistability depends extremely on the memristor initial condition and the system parameters. It is shown that the nonlinear dynamics of the financial chaotic system can be significantly changed by changing the values of time delays.

Results of A. Hadjimichael et al. show that classical assumptions for fisheries management can yield severe instabilities in the quantified views of socioecological tradeoffs, making their ability to inform stakeholder preferences questionable. The complex ecological interactions implied by different parameterizations of such systems yield highly complex and nonlinear dynamic properties with multiple distinct basins of attraction. The authors show that small changes in the deeply uncertain representations of predator-prey systems can fundamentally shift their dynamics and the validity of candidate management strategies for harvest. Insights from this study highlight the importance of ensuring models capture deep uncertainties, as well as a breadth of financial and ecological criteria, when searching for robust management options for resilient fisheries.

A class of two-parameter mixed-mode oscillation with time delay under the action of amplitude modulation is studied by Y. Qian and W. Meng. The investigation is from four aspects. Firstly, a parametric equation is considered as a slow variable. By the time-history diagram and phase diagram, the authors can find that the system generates a cluster discovery image. Secondly, the Euler method is used 
to discrete the system and obtain the discrete equation. Thirdly, the dynamic characteristics of the system at different time scales are discussed when the ratio of the natural frequency and the excitation frequency of the system is integer and noninteger. Fourthly, the authors discuss the influence of time delay on the discovery of clusters of this kind of system. The research shows that the time lag does not interfere with the influence of the cluster image, but the dynamics of the upper and lower parts of the oscillation in each period will be delayed. So, they can improve peak performance by adjusting the time lag and obtain the desired peak. Finally, the authors explore the multistate dynamic response of a two-dimensional nonautonomous Duffing system with higher order. According to the bifurcation diagram and the time-history curve, bistable state will appear in the system within the critical range. With the gradual increase of parameters, the chaotic attractor will suddenly disappear which will lead to the destruction of the bistable state.

F. A. Rihan et al. study the dynamics of a two-prey onepredator system, where the growth of both prey populations is subject to Allee effects, and there is a direct competition between the two-prey species having a common predator. Two discrete time delays $\tau_{1}$ and $\tau_{2}$ are incorporated into the model to represent the reaction time of predators. Sufficient conditions for local stability of positive interior equilibrium and existence of Hopf bifurcations in terms of threshold parameters $\tau_{1}^{*}$ and $\tau_{2}^{*}$ are obtained. A Lyapunov functional is deducted to investigate the global stability of positive interior equilibrium. Sensitivity analysis to evaluate the uncertainty of the state variables to small changes in the Allee parameters is also investigated. Presence of Allee effect and time delays in the model increases the complexity of the model and enriches the dynamics of the system. Some numerical simulations are provided to illustrate the effectiveness of the theoretical results. The model is highly sensitive to small changes in Allee parameters at the early stages and with low population densities, and this sensitivity decreases with time.

The article by S. M. Boulaaras et al. deals with the study of the existence of weak positive solutions for sublinear Kirchhoff elliptic systems with the zero Dirichlet boundary condition in the bounded domain $\Omega \subset \mathbb{R}^{N}$ by using the subsupersolution method.

The article by Y. Bouizem et al. deals with the study of the existence of weak positive solutions for a new class of the system of elliptic differential equations with respect to the symmetry conditions and the right-hand side which has been defined as multiplication of two separate functions by using the sub-supersolution method.

By introducing a flux-controlled memristor model with absolute value function, a 5D multistable four-wing memristive hyperchaotic system (FWMHS) with linear equilibrium points is proposed in the article by F. Yu et al. The dynamic characteristics of the system are studied in terms of the equilibrium point, perpetual point, bifurcation diagram, Lyapunov exponential spectrum, phase portraits, and spectral entropy. This system is of the group of systems that have coexisting attractors. In addition, the circuit implementation scheme is also proposed. Then, a secure communication scheme based on the proposed $5 \mathrm{D}$ multistable FWMHS with disturbance inputs is designed. Based on parametric modulation theory and Lyapunov stability theory, synchronization and secure communication between the transmitter and the receiver are realized, and two message signals are recovered by a convenient robust high-order sliding mode adaptive controller. Through the proposed adaptive controller, the unknown parameters can be identified accurately, the gain of the receiver system can be adjusted continuously, and the disturbance inputs of the transmitter and the receiver can be suppressed effectively. Thereafter, the convergence of the proposed scheme is proven by means of an appropriate Lyapunov functional, and the effectiveness of the theoretical results is testified via numerical simulations.

The article by S. S. Askar and A. Al-khedhairi studies the dynamic characteristics of triopoly models that are constructed based on a 3-dimensional Cobb-Douglas utility function. The article presents two parts. The first part introduces a competition among three rational firms on which their prices are isoelastic functions. The competition is described by a 3-dimensional discrete dynamical system. The authors examine the impact of rationality on the system's steady-state point. The stability/instability of this point is illustrated. Numerically, the authors give some global analysis of the Nash point and its stability. The second part deals with heterogeneous scenarios. It consists of two different models. In the first model, the authors assume that one competitor adopts the local monopolistic approximation mechanism (LMA), while the other opponents are rational. The second model assumes two heterogeneous players with the LMA mechanism against one rational firm. Studies show that the stability of the NE point of those models is not guaranteed. Furthermore, simulation shows that when firms behave rational with symmetric costs, the stability of the NE point is achievable.

The article by $\mathrm{H}$. Wu et al. presents a novel and simple three-dimensional (3D) chaotic system by introducing two sine nonlinearities into a simple 3D linear dynamical system. The presented sine system possesses nine equilibrium points consisting of five index-2 saddle foci and four index-1 saddle foci which allow the coexistence of various types of disconnected attractors, also known as multistability. The coexisting multiple attractors are depicted by the phase plots and attraction basins. Coexisting bifurcation modes triggered by different initial values are numerically simulated by two-dimensional bifurcation and complexity plots under two sets of initial values and one-dimensional bifurcation plots under three sets of initial values, which demonstrate that the abundant coexisting multiple attractors' behaviors in the presented sine system are related not only to the system parameters but also to the initial values. A simulation-oriented circuit model is synthesized, and PSIM (power simulation) screen captures well validate the numerical simulations. 


\section{Conflicts of Interest}

The editors declare that they have no conflicts of interest.

\section{Acknowledgments}

The editorial team would like to express their appreciation to all authors for their valuable contributions. In addition, the editors would like to thank the Complexity Journal's Editorial Board for their valuable help and support regarding this special issue.

Viet-Thanh Pham

Sundarapandian Vaidyanathan Tomasz Kapitaniak 